\title{
Artritis séptica de la articulación temporomandibular como complicación de una celulitis facial: reporte de un caso
}

\section{Septic arthritis of the temporomandibular joint after complication of facial cellulitis: case report}

\section{Resumen}

La infección del cóndilo mandibular, artritis séptica, es una enfermedad caracterizada por dolor, fiebre, edema y disminución funcional de la articulación temporomandibular. Predomina en hombres adultos y su etiología incluye distintos factores: enfermedades sistémicas, autoinmunes, trauma local y diseminación de infecciones en la región de cabeza y cuello. Se presenta un caso clínico de artritis séptica en articulación temporomandibular como complicación de una celulitis facial infecciosa. El tratamiento consistió en antibioterapia y artrocentesis seriadas con el objetivo de realizar un aseo intraarticular, acompañado de la movilización temprana de la articulación, idealmente con el apoyo de un equipo de kinesiología. Además, son muy importantes los controles periódicos y de larga data para poder obtener un mejor resultado clínico en el paciente, controlar en el largo plazo, minimizando los riesgos de presentar disminución en la dinámica mandibular y/o anquilosis de la articulación.

Palabras clave: Artritis séptica; Articulación temporomandibular; Artrocentesis (fuente: DeCS BIREME).

\begin{abstract}
Mandibular condyle infection, septic arthritis, is a disease characterized by pain, fever, edema, and functional decrease of the temporomandibular joint. It predominates in adult men and its etiology includes different factors: systemic diseases, autoimmune diseases, local trauma and spread of infections in the head and neck region. A clinical case of septic arthritis in the temporomandibular joint as a complication of infectious facial cellulitis is presented. Treatment consisted of antibiotic therapy and serial arthrocentesis with the aim of performing an intra-articular cleaning, accompanied by early mobilization of the joint, ideally with the support of a kinesiology team. In addition, long-term and periodic controls are very important in order to obtain a better clinical result in the patient, control in the long term, minimizing the risks of presenting a decrease in mandibular dynamics and / or ankylosis of the joint.
\end{abstract}

Keywords: Septic arthritis; Temporomandibular joint; Arthrocentesis (source: MeSH NLM).

\section{Caso Clínico}

Nicole Sabelle ${ }^{1, a}$, Valentina Vergara ${ }^{1,3, a}$,

Marcos Rodrigues ${ }^{3, b}$, Marcelo Mardones ${ }^{1,2,3,4, b, c,}$, Rodrigo Bravo 1,2,3,4,0

${ }^{1}$ Universidad de Chile, Santiago, Chile.

${ }^{2}$ Hospital San José, Servicio de Cirugía, Equipo de Cirugía y Traumatología Bucal y Maxilofacial, Santiago, Chile. ${ }^{3}$ Universidad de Chile, Facultad de Odontología, Departamento de Cirugía y Traumatología Bucal y Maxilofacial, Santiago, Chile.

${ }^{4}$ Clínica Las Condes, Departamento de Cirugía Maxilofacial, Santiago, Chile.

a Cirujano Dentista.

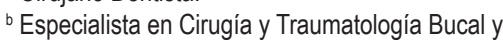

Máxilo-Facial.

${ }^{c}$ Magíster en Ciencias Biológicas.

\section{Correspondencia:}

Rodrigo Bravo: rbravo14@gmail.com

Olivos 943, Independencia, Santiago, Chile.

ORCID: 0000-0002-3480-1215

\section{Coautores:}

Nicole Sabelle: nicole.sabelle@gmail.com ORCID: 0000-0001-6765-2263

Valentina Vergara: valentina.vergara2003@gmail.com ORCID: 0000-0003-0301-075X

Marcos Rodrigues: marcosrodriguezurbina@gmail.com ORCID: 0000-0002-9610-8360

Marcelo Mardones: drmardones@gmail.com

ORCID: 0000-0003-4936-3294

\section{Editora:}

Marieta Petkova-Gueorguieva

Universidad Nacional Mayor de San Marcos, Perú.

Conflicto de intereses: $\sin$ conflicto de intereses.

Fuente de financiamiento: autofinanciado.

Recibido: $22 / 06 / 20$

Aceptado: $22 / 09 / 20$

Publicado: $16 / 11 / 20$ 


\section{Introducción}

La infección del cóndilo mandibular, conocida como artritis séptica, es una enfermedad poco común caracterizada por dolor, fiebre, edema y disminución funcional de la articulación temporomandibular (ATM) ${ }^{1}$. Predomina en hombres adultos y su etiología incluye distintos factores como: enfermedades sistémicas, autoinmunes, trauma local y diseminación de infecciones en la región de cabeza y cuello ${ }^{2}$. La celulitis facial de origen infeccioso es una de las patologías más comunes en esta región, que va de un $4-24 \%$ de todos los casos de celulitis reportados ${ }^{3}$. Algunos estudios reportan una asociación con causas iatrogénicas como la exodoncia de terceros molares y la distracción osteogénica ${ }^{4}$. La patogénesis de la artritis séptica en el cóndilo mandibular se puede deber a una diseminación hematológica de los microorganismos o la extensión directa de una infección contigua. Los microorganismos que se han visto asociados son Staphylococcus, Neisseria gonococcus, Streptococcus y Haemophilus influenzae 5 . Si bien, no existe consenso para establecer un criterio diagnóstico y un tratamiento para esta infección del cóndilo mandibular, en la mayoría de los estudios se reporta el uso de aspiración, análisis de fluidos, exámenes de laboratorio, imágenes y exploración clínica para su diagnóstico y tratamiento. Por otro lado, dentro de las opciones terapéuticas se indica antibioterapia por periodos prolongados, en conjunto con otras opciones de tratamiento como artrocentesis, artroscopía y condilectomía ${ }^{5-7}$.

El objetivo de este reporte es presentar un caso de artritis séptica en cóndilo mandibular derecho posterior, en un hombre de 26 años, con un cuadro de celulitis infecciosa de espacios infratemporal y pterigomandibular derecho como complicación de una pericoronaritis del tercer molar ipsilateral, porque nos parece relevante abordar las opciones de tratamiento, en este caso artrocentesis y terapia antibiótica, además de mostrar la importancia del trabajo de equipo interdisciplinario.

\section{Reporte del caso}

Paciente hombre de 26 años de edad, estudiante. Sin antecedentes mórbidos ni alergias, con hábitos de consumo de marihuana y cocaína de forma ocasional. Ingresa al servicio de urgencias del Hospital San José (HSJ) por aumento de volumen en la región perimandibular derecha. Refiere trismus, dolor 5/10 en escala EVA y sensación febril. Al examen físico general presenta fiebre de $39^{\circ} \mathrm{C}$, nódulos linfáticos submandibulares palpables y compromiso del estado general. El examen físico segmentario constata limitación en la dinámica mandibular y aumento de volumen submandibular. Al examen intraoral se observa alveolo de diente 4.7 en proceso de cicatrización, diente 4.8 con capuchón pericoronario eritematoso y exudado purulento, dolor a la palpación en espacio pterigomandibular derecho, fondo de vestibulo y piso de boca sin compromiso infeccioso. $\mathrm{La}$ tomografía computarizada (TC) con contraste presenta espacio laterofaríngeo derecho levemente desplazado, área hipodensa en espacio pterigomandibular e infratemporal derecho (Figura 1). Sin evidencia de espacios abscedados. Paciente tomo conocimiento del caso y firmó consentimiento del tratamiento propuesto y el registro de su caso clínico.

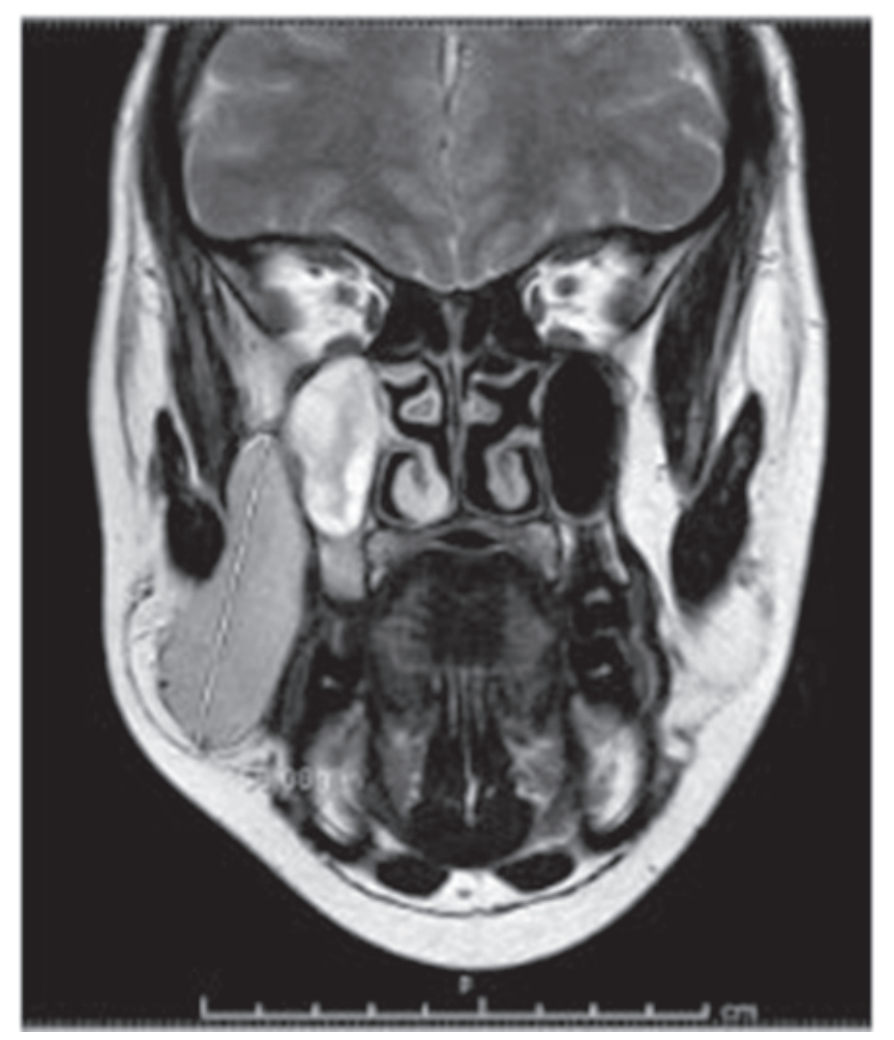

Figura 1. Imagen TC con medio contraste, se observa colección en región pterigomandibular derecha 
Se indicó tratamiento antibiótico con 2 gramos de ceftriaxona cada 24 horas y 600 miligramos de clindamicina cada 8 horas endovenosa por 1 semana, además se realizó aseo quirúrgico de la zona y extracción del diente 4.8. Su evolución fue favorable, por lo que el paciente fue dado de alta, una vez completado el tratamiento.

$\mathrm{Al}$ mes, paciente ingresó nuevamente al servicio de urgencias del HSJ por dolor e impotencia funcional. Refirió sensación febril, dolor $6 / 10$ en escala EVA, trismus y compromiso del estado general por lo que fue hospitalizado nuevamente. Al examen físico se observó asimetría facial por aumento de volumen en la región preauricular derecha de 1 semana de evolución de consistencia firme, indurado, con límites mal definidos, de 5x6 centímetros de diámetro, sin cambios de coloración en la piel, acompañado de limitación de apertura bucal de $20 \mathrm{~mm}$, dolor a la palpación y sin alteraciones neurológicas. No se palpaban nódulos linfáticos en la región de cuello y submandibular (Figura 2). El paciente manifestó pérdida de peso de 6 kilogramos en menos de 1 mes. Los exámenes de laboratorio arrojaron resultado de parámetros inflamatorios alterados, con una leucocitosis y velocidad de sedimentación aumentada.

Se solicitó TC actualizada en la cual se observó áreas hipodensas en cóndilo mandibular derecho (Figura 3), compatible con artritis séptica de ATM derecha. Tras

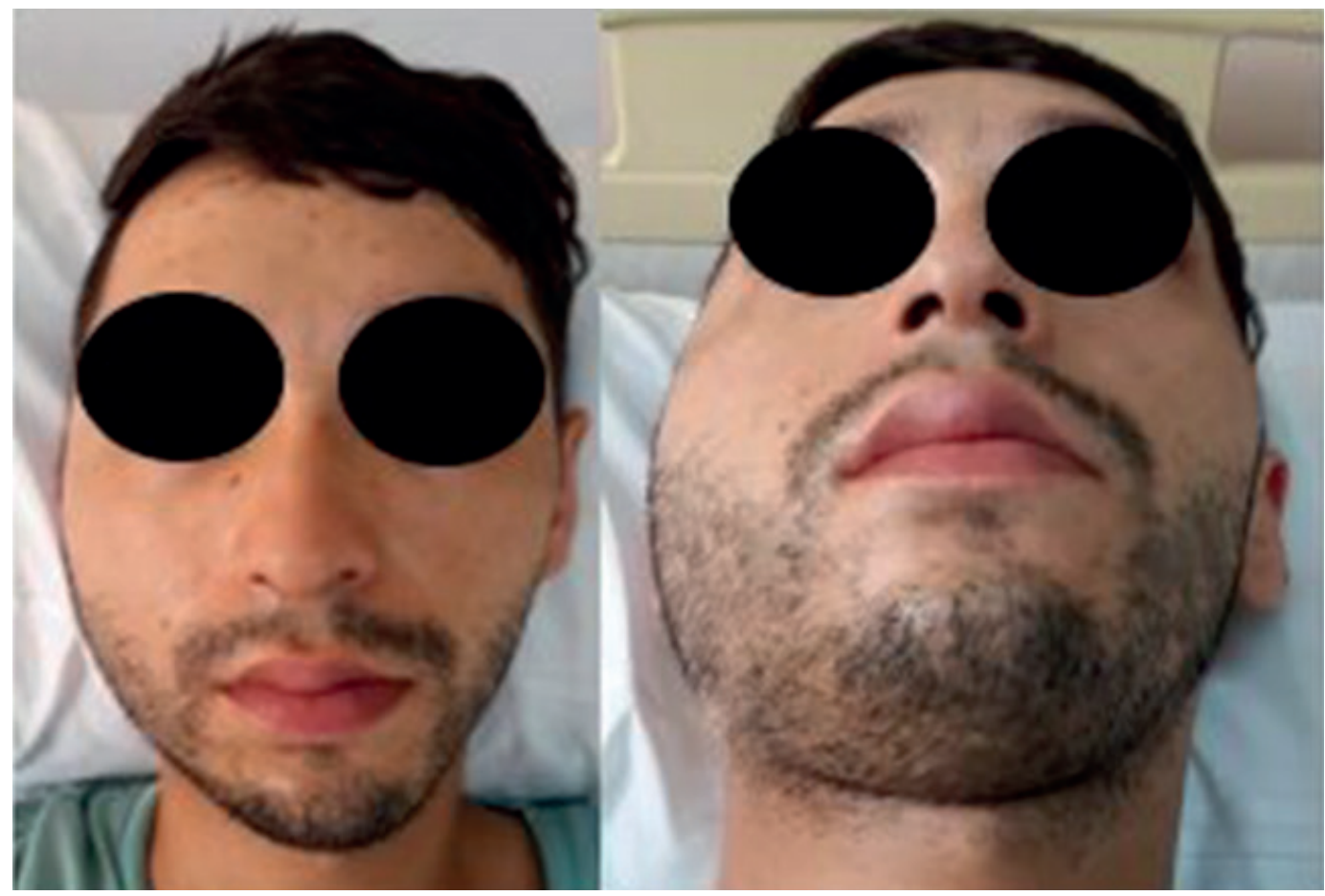

Figura 2. Imagen clínica de la segunda consulta, se observa aumento volumen región preauricular derecha

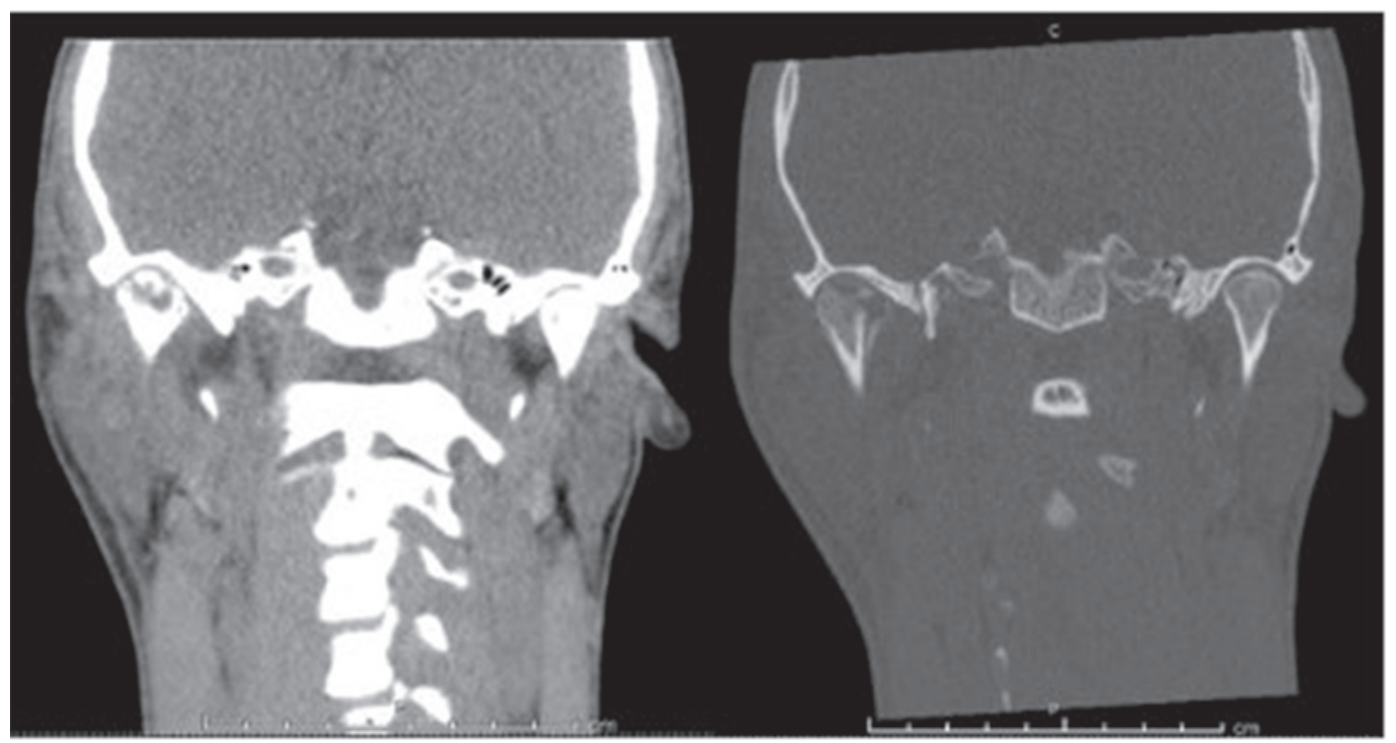

Figura 3. Imagen TC de reingreso hospitalario al mes, se observa erosión cortical ósea severa en cabeza de cóndilo derecho 
evaluación por infectología, se inició tratamiento endovenoso con piperacilina y tazobactam 4,5 gramos cada 8 horas en infusión prolongada por 14 días y como tratamiento quirúrgico se realizaron dos artrocentesis diferidas por una semana, con el objetivo de realizar un aseo quirúrgico intra articular. En la primera intervención se tomó cultivo del líquido sinovial que arrojo resultado negativo. El protocolo utilizado en la artrocentesis fue con dos punciones, una en receso posterior articular donde se realizó maniobra de pumping, luego una segunda punción en receso anterior articular para hacer circuito, lavado articular con solución ringer lactato 100 cc e infiltración final con betametasona $4 \mathrm{mg}$ intraarticular. Al completar antibioterapia paciente presenta una mejoría de los parámetros inflamatorios y funcionales, sin signos de infección por lo que se otorgó el alta a los 14 días con indicación de amoxicilina con ácido clavulánico por 3 meses. Se controló al mes, mostrando una evolución favorable, oclusión estable, buena dinámica mandibular y apertura bucal.

Se complementó con terapia kinésica, ya que de esta forma podemos ayudar a que la recuperación sea más rápida mitigando el dolor, reeducando la musculatura y evitando la posibilidad de una anquilosis. El protocolo empleado por el servicio de Kinesiología consistió en realizar tratamiento para el dolor con crioterapia, electroterapia y electroanalgesia, tratamiento para el edema con drenaje linfático manual y activación de musculatura orofacial en contracciones isométricas. Para la hipomovilidad se realizó distracción articular, deslizamiento medial y traslación. Para la reeducación muscular se restableció la dinámica muscular normal, ejercicios de control motor y fortalecimiento. A los 3 meses se realizó TC de control, mostrando signos de reparación ósea sin artritis séptica activa (Figura 4).

\section{Discusión}

En general, la artritis séptica en la ATM, es una enfermedad poco común, con solo 40 casos reportados en los últimos 80 ańos ${ }^{1}$. La artritis séptica en el resto del cuerpo afecta principalmente la rodilla en $50 \%$, y en menos porcentaje en orden decreciente a cadera, hombro, codo y el resto de las articulaciones del cuerpo, donde podríamos incluir a la $\mathrm{ATM}^{8}$. La artritis séptica en el cóndilo mandibular puede ser producida por contaminación vía hematógena o por contigüidad de proceso infeccioso ${ }^{9,10}$. El diagnóstico oportuno junto con el tratamiento de forma rápida son importantes para obtener un mejor resultado clínico y prevenir complicaciones como destrucción de la cápsula, adhesiones, anquilosis u osteomielitis ${ }^{11,12}$. En este caso se sospechó que el cuadro podría haber avanzado a una osteomielitis mucho más severa, sin embargo, el paciente presentó una evolución favorable. En general, los signos y síntomas que se han visto asociados a estos casos son fiebre, trismus y aumento de volumen preauricular, todos presentes en nuestro paciente ${ }^{1}$.

Una complicación es que la sintomatología inicial de la artritis séptica se podría confundir con otras alteraciones de la ATM como degeneración interna, condromatosis sinovial o artritis reumatoide. Muchas veces los cambios en el hueso no se observan hasta que el proceso ya está en su fase más aguda, por lo cual, es importante el estudio

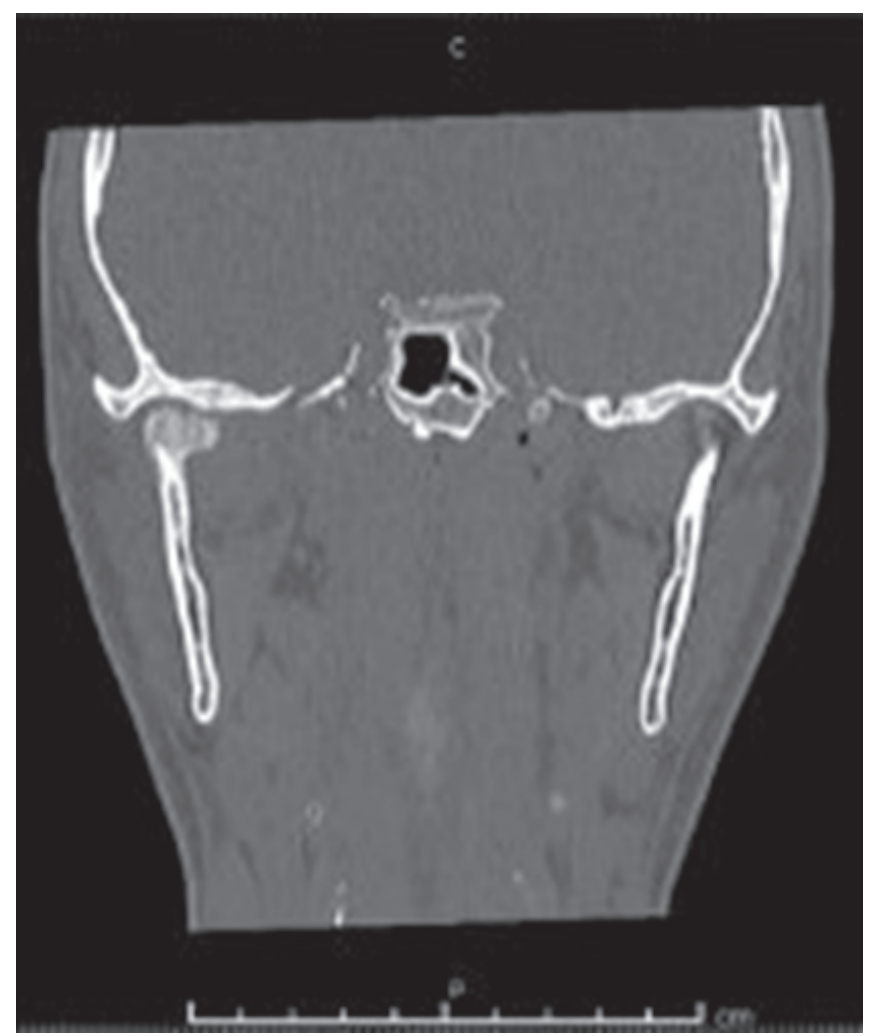

Figura 4. Imagen TC control 3 meses. Se observa signos de reparación ósea en cortical de cabeza de cóndilo derecho 
de la articulación con imágenes. La resonancia magnética es de utilidad para observar el compromiso de estructuras blandas articulares y la tomografía para ver el compromiso del tejido óseo. En este caso, la TC fue suficiente para poder confirmar la sospecha diagnóstica, ya que este estudio nos permite demostrar la erosión de la cortical o pérdidas de la cortical ósea, osteomielitis y/o anquilosis en la articulación ${ }^{13}$.

No existe evidencia suficiente para determinar cuál es el protocolo de tratamiento ideal, pero dentro de las intervenciones que se describen, se menciona la antibioterapia como tratamiento único o complementada con la artrocentesis, artroscopía o la condilectomía ${ }^{5,7}$. El tratamiento antibiótico empírico debe ser iniciado inmediatamente y reajustado una vez que se tenga el resultado de los análisis de laboratorio ${ }^{6,14}$. Los microorganismos que se han asociado habitualmente a la artritis séptica son Staphylococcus aureus, Streptococcus species, Pseudomonas aeruginosa, Escherichia coli, Neisseria y Haemophilus influenzae ${ }^{15}$. Aseo intraarticular y descompresión de la articulación también se describen como tratamientos esenciales para eliminar el contenido inflamatorio y permitir el estudio de los microorganismos presentes en el líquido sinovial para poder iniciar la terapia antibiótica específica. En este caso, el paciente fue sometido a tratamiento antibiótico empírico inicial y luego reajustado por el equipo de infectología del hospital. Con respecto al resultado negativo del cultivo, se puede explicar por la terapia antibiótica empírica instaurada inicialmente, lo cual podría haber modificado la flora al momento de la aspiración ${ }^{1}$. Por otro lado, es posible utilizar la artroscopía para permitir una visualización más directa de la articulación permitiendo lavados adicionales, lisis de las adhesiones y debridamientos ${ }^{5}$. En nuestro reporte, debido al rápido inicio del tratamiento la respuesta a la artrocentesis fue óptima, siendo innecesario recurrir a la artroscopía.

La artritis séptica de la ATM debe ser considerada como complicación de cuadros sépticos. Al no existir un protocolo único en el manejo de esta patología es imprescindible el examen físico, TC con contraste y estudios de laboratorio para poder realizar de forma oportuna el tratamiento, que puede ser basado en antibioterapia, aseos intraarticulares y movilización temprana de la articulación a través de kinesioterapia. Importantes son los controles periódicos de larga data para asegurar el cese de la enfermedad y obtener un mejor resultado clínico en el paciente, minimizando los riesgos de presentar alteraciones en funcionalidad articular y masticatoria.

\section{Referencias bibliográficas}

1. Cai XY, Yang C, Zhang ZY, Qiu WL, Chen MJ, Zhang SY. Septic arthritis of the temporomandibular joint: a retrospective review of 40 cases. J Oral Maxillofac Surg. 2010;68(4):731-8. DOI: 10.1016/j.joms.2009.07.060.

2. Baur DA, Altay MA, Flores-Hidalgo A, Ort Y, Quereshy FA. Chronic osteomyelitis of the mandible: diagnosis and management--an institution's experience over 7 years. J. Oral Maxillofac Surg. 2015;73(4):655-65. DOI:10.1016/j.joms.2014.10.017
3. Rath E, Skrede S, Mylvaganam H, Bruun T. Aetiology and clinical features of facial cellulitis: a prospective study. Infect Dis (Lond). 2018;50(1):27-34. DOI:10.10 80/23744235.2017.1354130.

4. Wang R, Cai Y, Zhao YF, Zhao JH. Osteomyelitis of the condyle secondary to pericoronaritis of a third molar: a case and literature review. Aust Dent J. 2014;59: 372374. DOI: 10.1111/adj.12186.

5. Sembronio S, Albiero AM, Robiony M, Costa F, Toro C, PolitI M. Septic arthritis of the temporomandibular joint successfully treated with arthroscopic lysis and lavage: case report and review of the literature. Oral Surg Oral Med Oral Pathol Oral Radiol Endod. 2007;103:e1-e6. DOI:10.1016/j.tripleo.2006.08.028.

6. Mathews CJ, Kingsley G, Field M, et al. Management of septic arthritis: a systematic review. Ann Rheum Dis. 2007;66(4):440-445. DOI: 10.1136/ard.2006.058909

7. Cai XY, Yang C, Chen MJ, Zhang SY, Yun B. Arthroscopic management of septic arthritis of temporomandibular joint. Oral Surg Oral Med Oral Pathol Oral Radiol Endod. 2010;109(1):24-30. DOI:10.1016/j.tripleo.2009.08.007.

8. Kaandorp CJ, Dinant HJ, van De Laar MA, Moens HJ, Prins AP, Dijkmans BA. Incidence and sources of native and prosthetic joint infection: a community based prospective survey. Ann Rheum Dis. 1997;56:470-5. DOI: 10.1136/ard.56.8.470.

9. Yang SW, Cho JY, Kim HM. Septic arthritis of the temporomandibular joint: a case report. J Korean Assoc Oral Maxillofac Surg. 2016;42(4):227-230. DOI: 10.5125/ jkaoms.2016.42.4.227.

10. Klüppel LE, Bernabé FB, Primo BT, Stringhini DJ, Da Costa DJ, Rebellato NL et al. Septic arthritis of the temporomandibular joint. J Craniofac Surg. 2012;23(6):17521754. DOI:10.1097/SCS.0b013e3182646061.

11. Amos MJ, Patterson AR, Worrall SF. Septic arthritis of the temporomandibular joint in a 6 -year-old child. Br J Oral Maxillofac Surg. 2008;46(3):242-243. DOI:10.1016/j. bjoms.2007.04.019.

12. Yew CC, Rahman SA, Alam MK. Temporomandibular joint ankylosis in a child: an unusual case with delayed surgical intervention. BMC Pediatr. 2015;15:169. DOI: 10.1186/s12887-015-0495-4.

13. Trimble LD, Schoenaers JA, Stoelinga PJ. Acute suppurative arthritis of the temporomandibular joint in a patient with rheumatoid arthritis. J Maxillofac Surg. 1983;11(2):92-95. DOI:10.1016/s03010503(83)80022-8.

14. Margaretten ME, Kohlwes J, Moore D, Bent S. Does this adult patient have septic arthritis?. JAMA. 2007;297(13):1478-1488. DOI:10.1001/ jama.297.13.1478.

15. Chaves Netto HD, Nascimento FF, Chaves Md, Chaves LM, Negreiros Lyrio MC, Mazzonetto R. TMJ ankylosis after neonatal septic arthritis: literature review and two case reports. Oral Maxillofac Surg. 2011;15(2):113-119. DOI:10.1007/s10006-010-0210-4. 
